\title{
Redundancy Elimination on RDF Graphs in the Presence of Rules, Constraints, and Queries ${ }^{\star}$
}

\author{
Reinhard Pichler ${ }^{1}$, Axel Polleres ${ }^{2}$, Sebastian Skritek ${ }^{1}$, and Stefan Woltran ${ }^{1}$ \\ 1 Technische Universität Wien, \{pichler, skritek, woltran\}@dbai.tuwien.ac.at \\ 2 DERI, National University of Ireland, Galway, axel.polleres@deri.org
}

\begin{abstract}
Based on practical observations on rule-based inference on RDF data, we study the problem of redundancy elimination on RDF graphs in the presence of rules (in the form of Datalog rules) and constraints, (in the form of so-called tuple-generating dependencies), and with respect to queries (ranging from conjunctive queries up to more complex ones, particularly covering features of SPARQL, such as union, negation, or filters). To this end, we investigate the influence of several problem parameters (like restrictions on the size of the rules, the constraints, and/or the queries) on the complexity of detecting redundancy. The main result of this paper is a fine-grained complexity analysis of both graph and rule minimisation in various settings.
\end{abstract}

\section{Introduction}

The Semantic Web promises to enable computers to gather machine readable meta-data in the form of RDF statements published on the Web and make inferences about these statements by means of accompanying standards such as RDFS and OWL2. While complete OWL2 reasoning is hard - and in many cases even inappropriate for Web data [1] - (incomplete) rule-based inference is becoming quite popular and supported by many RDF Stores and query engines: frameworks like GiaBATA [2], Jena, Sesame, OWLIM, ${ }^{3}$ etc. allow for custom inference on top of RDF Stores, supporting different rule-based fragments of RDFS and OWL. Several such fragments have been defined in the literature, such as $\rho \mathrm{DF}$ [3], DLP [4], OWL ${ }^{-}$[5], ter Horst's $\mathrm{pD}^{*}$ [6], or SAOR [7], and - more recently - the W3C standardised OWL2RL, a fragment of OWL implementable purely in terms of rule-based inference [8]. All these fragments have in common that they are implementable by simple Datalog-like rules over RDF. As an example, let us take (1) the sub-property rule from RDFS [9, Section 7.3, rule rdfs7], rules (2)-(5) from OWL2RL [9, Section 4.3, rules prp-inv1,prp-symp,prp-spo2] representing inverse properties, symmetric properties, and property chains: ${ }^{4}$

* Preliminary results have been presented at the Alberto Mendelzon Workshop 2010.

3 cf. http://jena.sourceforge.net/, http://openrdf.org/, and http://ontotext.com/owlim/

${ }^{4}$ We disregard full URIs for common RDF terms, i.e., we just write e.g. inverse $O f$, for <http://www.w3.org/2002/07/owl\\#inverse0f>, name for <http://xmlns.com/foaf/0.1/name>, or creator for $\left\langle\right.$ http://purl.org/dc/elements/1.1/>, etc. Further, $\left(P_{1} \ldots P_{n}\right)$ in RDF is short for a fresh variable $X$ plus additional triples $X$ first $P_{1} . X_{1}$ rest $X_{2} . \ldots X_{n}$ first $P_{n} \cdot X_{n}$ rest nil . using reserved terms first, rest, nil. 
(1) $\{\mathrm{S}$ P $0 . \mathrm{P}$ subProperty $O f \mathrm{Q} \cdot \operatorname{uri}(\mathrm{Q})\} \quad \Rightarrow\{\mathrm{S} Q \mathbf{O}\}$

(2) $\{\mathrm{S} P \mathrm{P} . \mathrm{P}$ inverse Of $\mathrm{Q} \cdot \operatorname{uri}(\mathrm{O}) \wedge \operatorname{uri}(\mathrm{Q})\} \Rightarrow\{0 \mathrm{QS}\}$

(3) $\{\mathrm{S} P \mathrm{P} . \mathrm{P}$ inverseOf $\mathrm{Q} \cdot \operatorname{blank}(\mathrm{O}) \wedge \operatorname{uri}(\mathrm{Q})\} \Rightarrow\{0 \mathrm{Q} \mathrm{S}\}$

(4) $\{$ S P O . P type SymmetricProperty . uri(0) $\} \Rightarrow\{0$ P S $\}$

(5) $\{$ S P O . P type SymmetricProperty . blank(0) $\} \Rightarrow\{$ O P S $\}$

(6) $\left\{\begin{array}{llllll}\mathrm{S} \mathrm{P}_{0} & \mathrm{O}_{1} \\ . & \ldots & \mathrm{O}_{n} & \mathrm{P}_{n} & \left.0 . \mathrm{P} \text { propertyChainAxiom }\left(\mathrm{P}_{0} \ldots \mathrm{P}_{n}\right)\right\} \Rightarrow\{\mathrm{S} \mathrm{P} & 0\end{array}\right\}$

Let $G_{D}$ be an RDF graph talking about authors and their publications:

(7) $G_{D}=\{<$ http://semanticweb.org/wiki/Pat_Hayes> made <http://www.w3.org/TR/rdf-mt/>. <http://semanticweb.org/wiki/Pat_Hayes> name "Patrick J. Hayes".

Moreover, let graph $G_{O}$ be part of the ontology defining the terms used in $G_{D}$ :

(10) $G_{O}=\{$ name subPropertyOf label.

(11) inverseOf type SymmetricProperty.

(12) made inverseOf maker.

(13) maker inverse Of made.

(14) creator propertyChainAxiom (maker label). $\}$

When storing the graph $G=G_{D} \cup G_{O}$ in an RDF Store that supports inference over rules (1)-(6), different questions of redundancy arise like if some statements may be deleted since they can be inferred by the rules. In our example, e.g. statement (9) as well as statement (13) may be deleted, since they could be reproduced by inference. Similarly, suppose that we transfer the graph $G=G_{D} \cup G_{O}$ to a "weaker" RDF Store that only supports rules (1)-(3). Then the question is if we thus loose any inferences. In fact, the answer is no. Interestingly enough, standard rule sets, such as OWL2RL are even known to be non-minimal [8, Section 4.3].

We thus want to be able to answer the general question about redundancy of both triples and rules. However, it is often important to limit the minimisation of RDF graphs in such a way that certain consistency conditions must be preserved. These consistency conditions can be expressed by means of constraints [10]. We shall restrict ourselves here to constraints in the form of so-called tuple-generating dependency $(t g d)$ constraints, which are a generalisation of the familiar foreignkey dependencies in the relational database world. Roughly speaking, a tgd may be viewed as a generalised rule "read" as constraint. So, for instance, if we read rules (4)-(5) as constraints, we could say that graph $G$ alone without rules satisfies these constraints, and likewise the closure of $G$ with respect to rules (1)(3) does. Tgd constraints can be more general than (Horn) rules in that they also allow otherwise unbound, existential variables in the head, possibly occurring in a larger conjunct. That is, tgds are - rather than rules - constraining queries (in the head) "triggered" by bindings coming from a query in the body; for instance, a constraint

\section{(15) $\{$ A made D $\} \Rightarrow\{$ A label N . D creator $\mathrm{N}\}$}

would hold only on graphs where everybody who made something also has a declared label and that label is also used to denote the creator. Note that constraint (15) holds on the closure of $G$ with respect to rule (1) but - as opposed to the constraint reading of (4)-(5) - not on $G$ alone. 
Next, we are interested in redundancy with respect to queries. This might be particularly relevant for RDF stores that expose a narrow SPARQL query interface. For instance, suppose that, in our example, we are interested only in completeness with respect to the query "SELECT ?D ?L \{ ?D maker ?M . ?M label ?L $\}$ " which is the SPARQL way of writing a conjunctive query:

(16) $\{$ D maker M . M label L $\} \rightarrow \operatorname{ans}(D, L)$

In this setting, both rules (3)-(6), as well as triples (9),(11),(13), and (14) can be dropped. Such redundancy elimination is not unique; for instance, keeping triples (11), (13), and rule (4) we could drop (12), still preserving completeness.

The primary goal of our work is a systematic complexity analysis of both graph and rule minimisation under constraints, as well as with respect to queries. To this end, we investigate the influence of several problem parameters (like restrictions on the size of the rules, constraints, and queries) on the complexity of detecting redundancy. A first important step in this investigation has been recently made by Meier [11]. He studied the following problem: Given a graph $G$, a set $\mathcal{R}$ of rules and a set $\mathcal{C}$ of tgds, can $G$ be reduced to a proper subgraph $G^{\prime} \subset G$, such that $G^{\prime}$ still satisfies $\mathcal{C}$ and the closure of $G^{\prime}$ under $\mathcal{R}$ coincides with the closure of $G$ under $\mathcal{R}$ ? For the special case that both the rules in $\mathcal{R}$ and the constraints in $\mathcal{C}$ have bounded size (referred to as b-boundedness), this problem was shown to be NP-complete in [11]. In this paper, we want to extend the work initiated in [11] and provide a much more fine-grained analysis of the complexity, e.g., by weakening or strengthening restrictions such as b-boundedness and by considering redundancy elimination that only preserves RDF entailment (rather than keeping the closure of the original graph under the original rules unchanged) and additionally considering redundancy with respect to queries.

We shall come up with a collection of complexity results, ranging from tractability to $\Sigma_{3}^{P}$-completeness. Additionally, we address the orthogonal problems of rule minimisation and the problem of reducing rules or triples without preserving completeness of the entire closure, but only ensuring that the answers to certain queries are preserved.

We shall also discuss further variations of the graph and rule minimisation problem. For instance, the rules and tgds in [11] do not allow variables in predicate positions, which is a severe restriction in the sense that many of the common RDF inferences rules are not covered (e.g., all except rules (4) and (5) above). We will not make this restriction, since it can be dropped without significant change of the complexity results.

Organisation of the paper and summary of results. In Section 2, we recall some basic notions and results. A conclusion and an outlook to future work are given in Section 7. Sections 3-6 contain the main results of the paper, namely:

- Graph Minimisation. In Section 3, we provide a comprehensive complexity analysis of the RDF graph minimisation problem, both when full reconstruction of the graph or only RDF entailment is required. We study various settings which result from different restrictions on the rules and/or tgds like restricting their size, considering them as fixed, omitting them, or imposing no restrictions at all. Our complexity results range from tractability to $\Sigma_{3}^{P}$-completeness.

- Rule Minimisation. In Section 4, we consider the problem of minimising the set of rules. We show that the problem of finding redundant rules with respect to 
a given RDF graph is NP-complete for b-bounded rules and not harder than $\Delta_{2}^{P}$ for arbitrary rules. Note that rule minimisation is closely related to the field of Datalog equivalence and optimisation. We therefore discuss how the large body of results in this area can be fruitfully applied to the problems studied here.

- Graph Minimisation w.r.t. Queries. In Section 5, we study how guaranteeing completeness only w.r.t. a given set of conjunctive queries (CQs) or unions of conjunctive queries (UCQs) influences the complexity for each of the above settings. Considering different restrictions on the size of the queries, hardness never exeeds $\Sigma_{3}^{P}$, but for some settings raises by two levels in the polynomial hierarchy compared to Section 3. Finally we extend our findings to the problem of rule minimisation. We shall also briefly touch on full SPARQL queries beyond unions of conjunctive queries.

- Problem Variations. In Section 6, we analyse the complexity of further problems which are either variations of or strongly related to the graph and rule minimisation problems mentioned above. For instance, rather than asking if an RDF graph contains redundant tuples, we consider the problem whether an RDF graph can be reduced below a certain size. We show that this problem is NP-complete also in those settings where the graph minimisation problem is tractable. We also discuss the effect of allowing blank nodes in predicate positions in the Datalog rules.

Due to lack of space, proofs are only sketched. While for most of the hardness proofs we only describe the idea of the reduction, membership proofs are either also informal or even omitted. All proofs are worked out in detail in [12].

\section{Preliminaries}

Let $U, B$, and $L$ denote pairwise disjoint alphabets for $U R I$ references, Blank nodes (or variables) and Literals, respectively. We denote unions of these sets simply by concatenating their names. ${ }^{5} \mathrm{An}$ RDF statement (or triple) is a statement of the form $(s, p, o) \in U B \times U \times U B L$, and an RDF graph is a set of triples. In this paper, we do not distinguish between variables and blank nodes, but just note that blank nodes/variables appearing in the data are understood to be existentially quantified within the scope of the whole RDF graph they appear in. We write elements from $B(U)$ as alphanumeric strings starting with an upper case letter (lower case letter or number), elements from $L$ as quoted strings, and - inspired by the common Turtle [13] syntax - RDF statements as white-space separated triples and RDF graphs as '.' separated lists of triples in curly braces.

It is convenient to define the notion of entailment between two RDF graphs via the interpolation lemma from $[9$, Section 2] rather than in a model-theoretic way: an RDF graph $G_{1}$ entails $G_{2}$, written $G_{1} \models G_{2}$ if a subgraph of $G_{1}$ is an instance of $G_{2}$, that is, if there exists a graph homomorphism, i.e., a blank node mapping $\mu: B \rightarrow U B L$ such that $\mu\left(G_{2}\right) \subseteq G_{1}$, where $\mu(G)$ denotes the graph obtained by replacing every variable $\mathrm{B} \in B$ with $\mu(\mathrm{B})$. A homomorphism $h^{\prime}$ is an extension of a homomorphism $h$ if $h^{\prime}(\mathrm{B})=h(\mathrm{~B})$ for all $\mathrm{B}$ on which $h$ is defined.

\footnotetext{
${ }^{5}$ In this paper, we use a slightly simplified notion of RDF compared to [9], e.g. not considering typed literals separately.
} 
Given $G_{1}, G_{2}$, deciding whether there exists a homomorphism $G_{2} \rightarrow G_{1}$ (thus also $G_{1} \models G_{2}$ ) is well known to be NP-complete.

We define a basic graph pattern (BGP) as a set of generalised triples $\left(s^{\prime}, p^{\prime}, o^{\prime}\right)$ $\in U B L \times U B L \times U B L$, a filter condition as a conjunct of the unary predicates uri $(\cdot)$, blank $(\cdot)$, literal $(\cdot)$ (denoting the unary relations $U, B$, and $L$, respectively). A filtered basic graph pattern (FBGP) is a BGP conjoined with a filter condition, the latter containing only variables already appearing in the BGP. Given an FBGP $P$, we write $B G P(P)$ and $F(P)$ to denote its components, i.e. its BGP and its filter condition, respectively.

We define an RDF tuple-generating dependency (tgd) constraint (or simply constraint) $r$ as Ante $\Rightarrow \mathcal{C}$ on, where the antecedent $\mathcal{A}$ nte is an FBGP and the consequent $\mathcal{C}$ on is a BGP. A constraint $\mathcal{A}$ nte $\Rightarrow \mathcal{C}$ on is a short-hand notation for the first-order formula $\forall \boldsymbol{x}(\mathcal{A}$ te $(\boldsymbol{x}) \rightarrow(\exists \boldsymbol{y}) \mathcal{C}$ on $(\boldsymbol{x}, \boldsymbol{y})$ ) (where $\boldsymbol{y}$ denotes the blank nodes occurring in $\mathcal{C}$ on only, while $\boldsymbol{x}$ are the remaining blank nodes) Hence, a constraint $\mathcal{A}$ te $\Rightarrow \mathcal{C}$ on is satisfied over an RDF graph $G$ if for each homomorphism on $\boldsymbol{x}$ mapping $\operatorname{BGP}(\mathcal{A}$ Ante $)$ to $G$, there exists an extension $h^{\prime}$ of $h$ to $\boldsymbol{y}$ s.t. $h^{\prime}(\mathcal{C}$ on $) \subseteq G$. To increase the readability, we will sometimes explicitly write out the quantifiers and variable vectors. $R D F$ rules (or simply rules), are syntactically restricted constraints, where all variables appearing in $\mathcal{C}$ on also appear in Ante (akin to the common notion of safety [14] in Datalog). In the following, we will call RDF rules with an empty filter condition Datalog rules. ${ }^{6}$ We define the closure of a graph $G$ with respect to a set $\mathcal{R}$ of rules, written $C l_{\mathcal{R}}(G)$ as usual by the least fix-point of the immediate consequence operator. For a given graph $G$ or a given set $\mathcal{R}$ of rules, we use $X_{G}, X_{\mathcal{R}}(X \in\{U, B, L\})$ to denote the subset of $U$ (resp. $B, L$ ) used in $G$, or $\mathcal{R}$, respectively.

A conjunctive query $(C Q)$ over an $\operatorname{RDF}$ graph $G$ is of the form $G_{q} \rightarrow$ ans $(\boldsymbol{X})$, where $G_{q}$ is an FBGP, ans is a distinguished predicate, and $\boldsymbol{X}$ is a vector of blank nodes. We refer to $G_{q}$ as the body of $q(\operatorname{bod} y(q))$, and to ans $(\boldsymbol{X})$ as the head of $q($ head $(q))$. A union of conjunctive queries (UCQs) is a set of CQs, all having the same head. The result of a CQ $q$ over some RDF graph $G$ is defined as the set $q(G)=\left\{(\boldsymbol{x}) \mid\right.$ for all $x_{i} \in \boldsymbol{x}: x_{i} \in U_{G} B_{G} L_{G} U_{q} L_{q}$, there exists a homomorphism $\tau: B_{q} \rightarrow U_{G} B_{G} L_{G}$ s.t. $\tau(\operatorname{bod} y(q)) \subseteq G$ and $\left.\boldsymbol{x}=\tau(\boldsymbol{X})\right\}$. The result of a UCQ is the union of the results of its CQs.

We say that a rule or constraint is $b$-bounded if both, antecedent and consequent contain at most $b$ triples. We say a conjunctive query $q$ is body-b-bounded if $\operatorname{body}(q)$ is b-bounded, and we denote $q$ as head-b-bounded if $|\boldsymbol{X}| \leq b$ for some constant $b$ (however, $\operatorname{body}(q)$ may be arbitrary). A set $\mathcal{Q}$ of (U)CQs is body-bbounded (resp. head-b-bounded) if every $q \in \mathcal{Q}$ is body-b-bounded (resp. head b-bounded). Finally, we write $[n]$ to denote the set $\{1, \ldots, n\}$.

\section{RDF Graph Minimisation}

In this section, we study the complexity of RDF graph minimisation. For different restrictions on the input parameters, the complexity varies between tractability and $\Sigma_{3}^{P}$-completeness. Formally, we consider the following two basic problems:

\footnotetext{
${ }^{6}$ In fact, we will for most parts of the paper only consider Datalog rules, but will revisit the extension to arbitrary RDF rules at the end of Section 6, concluding that this extension does not change any of our results.
} 


\begin{tabular}{|l|l|l|c|}
\hline & & MINI-RDF $^{\models}$ & MINI-RDF \\
\hline$(1)$ & $\mathcal{R}$ arb., $\mathcal{C}$ arb. & $\Sigma_{3}^{P}$-complete & $\Sigma_{3}^{P}$-complete \\
\hline$(2)$ & $\mathcal{R}$ arb., $\mathcal{C}$ bb & NP-complete & NP-complete \\
\hline$(3)$ & $\mathcal{R}$ arb., $\mathcal{C}$ fixed & NP-complete & NP-complete \\
\hline$(4)$ & $\mathcal{R}$ arb., $\mathcal{C}=\emptyset$ & NP-complete & NP-complete \\
\hline$(5)$ & $\mathcal{R}$ bb., $\mathcal{C}$ arb. & $\Sigma_{3}^{P}$-complete & $\Sigma_{3}^{P}$-complete \\
\hline$(6)$ & $\mathcal{R}$ bb, $\mathcal{C}$ bb & NP-complete & NP-complete $[11]$ \\
\hline$(7)$ & $\mathcal{R}$ bb, $\mathcal{C}$ fixed & NP-complete & NP-complete \\
\hline$(8)$ & $\mathcal{R}$ bb, $\mathcal{C}=\emptyset$ & NP-complete & in P \\
\hline$(9)$ & $\mathcal{R}$ fixed, $\mathcal{C}$ arb. & $\Sigma_{3}^{P}$-complete & $\Sigma_{3}^{P}$-complete \\
\hline$(10)$ & $\mathcal{R}$ fixed, $\mathcal{C}$ bb & NP-complete & NP-complete \\
\hline$(11)$ & $\mathcal{R}$ fixed, $\mathcal{C}$ fixed & NP-complete & NP-complete \\
\hline$(12)$ & $\mathcal{R}$ fixed, $\mathcal{C}=\emptyset$ & NP-complete & in P \\
\hline
\end{tabular}

Table 1. The complexity of MINI-RDF ${ }^{\vDash}$ and MINI-RDF $\subseteq$ w.r.t. input parameters ("bb" indicates the set to be b-bounded, and "arb." allows for arbitrary sets.)

Definition 1. Let MINI-RDF ${ }^{\models}(G, \mathcal{R}, \mathcal{C})$ be the following decision problem: INPUT: $R D F$ graph $G$, set $\mathcal{R}$ of $R D F$ rules, set $\mathcal{C}$ of tgds ( $G$ satisfies $\mathcal{C})$. QUESTION: Is there a $G^{\prime} \subset G$ s.t. $C l_{\mathcal{R}}\left(G^{\prime}\right) \models C l_{\mathcal{R}}(G)$ and $G^{\prime}$ satisfies $\mathcal{C}$ ?

Definition 2. Let MINI-RDF $\subseteq(G, \mathcal{R}, \mathcal{C})$ be the following decision problem [11]: INPUT: $R D F$ graph $G$, set $\mathcal{R}$ of $R D F$ rules, set $\mathcal{C}$ of tgds $(G$ satisfies $\mathcal{C})$.

QUESTION: Is there a $G^{\prime} \subset G$ s.t. $C l_{\mathcal{R}}(G)=C l_{\mathcal{R}}\left(G^{\prime}\right)$ and $G^{\prime}$ satisfies $\mathcal{C}$ ?

The MINI-RDF $\subseteq$ problem and the minimisation of $\mathrm{RDF}$ graphs via entailment aim at two kinds of redundancy elimination: In MINI-RDF $\subseteq$, triples which can be restored via the rules are considered as redundant while minimisation via entailment allows us to replace a graph $G$ by $\bar{G} \subset G$ if $\bar{G}=G$ holds, i.e. checks if $G$ is lean (see [15]). The MINI-RDF ${ }^{\models}(G, \mathcal{R}, \mathcal{C})$ problem combines these two approaches and thus yields the strongest redundancy criterion. Nevertheless, in most cases, its complexity is not higher than for MINI-RDF $\subseteq$ (see Theorem 1).

It is easy to see that the condition $C l_{\mathcal{R}}(G)=C l_{\mathcal{R}}\left(G^{\prime}\right)$ in Definition 2 is equivalent to $G \subseteq C l_{\mathcal{R}}\left(G^{\prime}\right)$. The following lemma shows that similarly, for MINI$\mathrm{RDF}^{\models}$, it is enough to show $C l_{\mathcal{R}}\left(G^{\prime}\right) \models G$ rather than $C l_{\mathcal{R}}\left(G^{\prime}\right) \models C l_{\mathcal{R}}(G)$.

Lemma 1. Let $G_{1}, G_{2}$ be $R D F$ graphs and $\mathcal{R}$ a set of rules. Then the following equivalence holds: $C l_{\mathcal{R}}\left(G_{2}\right) \models C l_{\mathcal{R}}\left(G_{1}\right) \Leftrightarrow C l_{\mathcal{R}}\left(G_{2}\right) \models G_{1}$.

Theorem 1. For MINI-RDF $\vDash$ and MINI-RDF $\subseteq$, the complexity w.r.t. different assumptions on the input (arbitrary, b-bounded, or fixed rule set; arbitrary, $b$ bounded, fixed, or no constraints) is as depicted in Table 1.
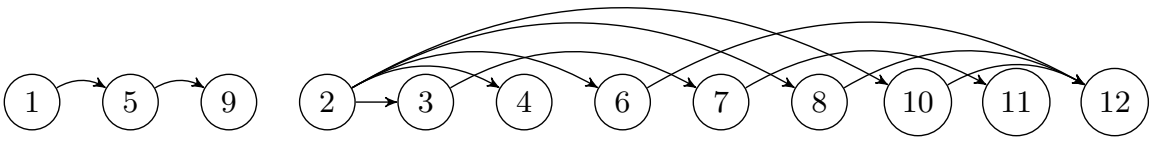

Fig. 1. Dependency graph: Numbers refer to lines in Table 1. An arrow from $A$ to $B$ means that $B$ is a special case of $A$. 
The following lemma justifies that we do not have to give an explicit completeness proof for each entry in Table 1, and points out a proof plan for Theorem 1.

Lemma 2. The graph in Figure 1 correctly describes the dependencies between the problems (identified by their line number) in Table 1, i.e.: If there is an arrow from $A$ to $B$, then $B$ is a special case of $A$.

Hence an arrow from $A$ to $B$ means that membership results for $A$ hold also for $B$, and that hardness results for $B$ apply also to $A$. Therefore, to prove Theorem 1, it suffices to show the membership for (1),(2),(8) and the hardness for $(4),(9),(11),(12)$. Due to lack of space, we only work out the hardness results for (9) and (11) (the latter only for MINI-RDF $\subseteq$ ). Before, we shortly discuss the membership results and give an intuition of why they are correct. All proofs are worked out in detail in the full paper [12].

The most general case, (1), can be solved by a guess and check algorithm that is allowed to use a $\Pi_{2}^{P}$ oracle for the checks. One has to guess: a subgraph $G^{\prime}$ of $G$, a sequence of rule applications on $G^{\prime}$, and for each rule application a homomorphism justifying that the rule is applicable. Note that $C l_{\mathcal{R}}\left(G^{\prime}\right) \subseteq$ $A D^{3}$ (with $A D=U_{G} U_{\mathcal{R}} B_{G} B_{\mathcal{R}} L_{G} L_{\mathcal{R}}$ ). Hence if considering all possible rule applications of length $|A D|^{3}$, one of them has to return $C l_{\mathcal{R}}\left(G^{\prime}\right)$. The most expensive check is to test if $G^{\prime}$ satisfies $\mathcal{C}$. However, it obviously fits into $\Pi_{2}^{P}$.

The following properties lead to the cases of lower complexity: If $\mathcal{R}$ is a b-bounded set, then $C l_{\mathcal{R}}\left(G^{\prime}\right)$ can be computed in polynomial time [11, Proposition 9] and if $\mathcal{C}$ is a b-bounded set, then testing if $G^{\prime}$ satisfies $\mathcal{C}$ is in PTIME [11, Proposition 3]. For the tractable cases, note that if $\mathcal{C}=\emptyset$, then not all subgraphs of $G$ have to be checked, but only those missing exactly one triple from $G$.

Lemma 3. The problems $\mathrm{MINI-RDF}{ }^{\models}(G, \mathcal{R}, \mathcal{C})$ and $\operatorname{MINI-RDF} \subseteq(G, \mathcal{R}, \mathcal{C})$, for fixed $\mathcal{R}$ and arbitrary $\mathcal{C}$, are $\Sigma_{3}^{P}$-hard.

Proof. $\Sigma_{3}^{P}$-hardness is shown by reduction from the well-known $\Sigma_{3}^{P}$-complete problem $\mathrm{QSAT}_{3}$, of which we only give an informal description here. Let an instance of $\mathrm{QSAT}_{3}$ be given by $F=\exists \boldsymbol{x}_{\mathbf{1}} \forall \boldsymbol{y}_{\mathbf{1}} \exists \boldsymbol{x}_{\mathbf{2}} \bigwedge_{i=1}^{n} C_{i}$, with $C_{i}=\left(l_{i, 1} \vee\right.$ $l_{i, 2} \vee l_{i, 3}$ ) (clearly, the restriction to 3-CNF is w.l.o.g.). The graph $G$ created contains on the one hand triples encoding truth assignments on clauses (e.g.

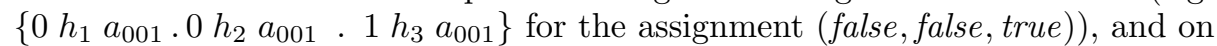
the other hand triples encoding the two possible truth assignments for variables (e.g. $\left\{v_{i} q_{1} a_{01} \cdot v_{i} q_{1} a_{10}\right\}$ for $x_{i} \in \boldsymbol{x}_{1}$ where $v_{i}$ is a new URI for each $x_{i}$ and the URI $a_{01}$ (resp. $a_{10}$ ) denotes that $x_{i}$ evaluates to false, hence $\neg x_{i}$ evaluates to true (resp. $x_{i}$ to true and $\neg x_{i}$ to false), together with further triples that allow us to actually refer to the truth value of $x_{i}\left(\right.$ resp. $\left.\neg x_{i}\right)$ ) under a selected truth assignment. The rules and constraints are chosen in such a way that (1) the triples encoding the truth assignment (false, false, false) for clauses must not be present in any valid subgraph $G^{\prime} \subset G,(2)$ for every $x_{i} \in \boldsymbol{x}_{\mathbf{1}}$ exactly one of the two triples encoding a truth assignment must be present in $G^{\prime}$ and (3) for all other variables, both triples have to remain in $G^{\prime}$. The restrictions imposed by $\bigwedge_{i=1}^{n} C_{i}$ are encoded in one big tgd, where every homomorphism from its antecedent to $G^{\prime}$ defines a truth assignment for $\boldsymbol{x}_{\boldsymbol{1}}$ and $\boldsymbol{y}_{1}$. Thereby for every valid $G^{\prime}$ all such homomorphisms define the same truth assignment on $\boldsymbol{x}_{1}$, hence the values for $x_{1}$ are determined by the selection of $G^{\prime}$. But every homomorphism defines a 
different truth assignment on $\boldsymbol{y}_{\mathbf{1}}$, and there exists exactly one homomorphism for each of the $2^{\left|\boldsymbol{y}_{1}\right|}$ truth assignments on $\boldsymbol{y}_{\mathbf{1}}$. The consequent of the tgd contains a representation of the literals in each clause $C_{i}$ and has the following property: for every homomorphism $h$ from the antecedent to $G^{\prime}$, there exists an extension of $h$ to a homomorphism $h^{\prime}$ from the consequent to $G^{\prime}$ iff this extension defines a truth assignment on $\boldsymbol{x}_{\mathbf{2}}$ such that the assignment on $\boldsymbol{x}_{\mathbf{1}}, \boldsymbol{y}_{\mathbf{1}}$ and $\boldsymbol{x}_{\mathbf{2}}$ maps the representations of the clauses onto the possible truth assignments for clauses present in $G^{\prime}$. As all triples encoding these truth assignments must be in $G^{\prime}$, except the ones for (false, false, false) which must not, such an extension for every homomorphism from the antecedent to $G^{\prime}$ implies that $F$ is valid.

Lemma 4. The problems $\operatorname{MINI-RDF} \subseteq(G, \mathcal{R}, \mathcal{C})$ and $\operatorname{MINI-RDF} \vDash(G, \mathcal{R}, \mathcal{C})$, where both, $\mathcal{R}$ and $\mathcal{C}$ are considered to be fixed, are NP-hard.

Proof. As NP-hardness of MINI-RDF ${ }^{\models}$ follows easily from the coNP-hardness of testing if $G$ is lean [15], we concentrate on MINI-RDF $\subseteq$ and prove its NPhardness by reduction from the 3-SAT problem. We fix the rules and tgds as

$$
\begin{aligned}
\mathcal{R}=\{ & \left.\left\{X^{\prime} \text { in } I . X \text { active } I\right\} \Rightarrow\left\{X^{\prime} \text { active } I\right\}\right\} \\
\mathcal{C}=\{ & \{X \text { active } I . X \text { in } J\} \Rightarrow\{X \text { active } J\} \\
& \left.\left\{X \text { clash } X^{\prime} . X \text { active } I . X^{\prime} \text { active } I^{\prime} . Y \text { in } J\right\} \Rightarrow\{Y \text { active } J\}\right\} .
\end{aligned}
$$

Now let an instance of 3-SAT be given by the formula $F=C_{1} \wedge \cdots \wedge C_{n}$, where $C_{i}=\left(l_{i, 1} \vee l_{i, 2} \vee l_{i, 3}\right)$ and the $l_{i, j}$ are literals. W.l.o.g., we assume that every variable appears negated and unnegated in $F$. Then we construct an RDF graph $G=\left\{l_{i, j}^{*}\right.$ in $\left.c_{i} \mid i \in[n], j \in[3]\right\} \cup\left\{l_{i, j}^{*}\right.$ active $\left.c_{i} \mid i \in[n], j \in[3]\right\} \cup\left\{x_{j}\right.$ clash $\bar{x}_{j} \mid$ $x_{j}$ in $\left.F\right\}$, where we introduce new URIs $c_{i}$ (for every clause $C_{i}$ ) and $x_{j}, \bar{x}_{j}$ (for every variable $x_{j}$ in $F$ ), and $l_{i, j}^{*}=x_{j}\left(\right.$ resp. $\left.\bar{x}_{j}\right)$ if $l_{i, j}=x_{j}\left(\right.$ resp. $\left.\neg x_{j}\right)$.

Intuitively, the triples in $G$ with predicate in encode the literals in $F$. If a triple with predicate active remains in the selected subgraph $G^{\prime}$ then the corresponding literal in $F$ is set to true. The triples with clash keep track of dual literals.

\section{Rule Minimisation}

In this section, we study the rule minimisation problem of RDF graphs. Although there is a huge amount of literature in the Datalog world addressing related problems (as query containment), the particular nature of the problems we study, requires a distinguished complexity analysis. Note that rules for RDF, when written as Datalog rules, have a fixed predicate arity of three, which makes problems computationally easier than in the general Datalog setting (see, e.g. [16]). Depending on whether we consider the Datalog rules as b-bounded or not, we obtain complexity results from NP-completeness to $\Delta_{2}^{P}$-membership. The rule minimisation problem is formally defined as follows. As the RDF graph remains unchanged, constraints are irrelevant here.

Definition 3. Let RDF-RULEMIN ${ }^{=}(G, \mathcal{R})$ be the following decision problem: INPUT: An RDF graph $G$ and a set $\mathcal{R}$ of $R D F$ rules. QUESTION: Does there exist $\mathcal{R}^{\prime} \subset \mathcal{R}$ s.t. $C l_{\mathcal{R}^{\prime}}(G) \models C l_{\mathcal{R}}(G)$ ? 
Definition 4. Let $\mathrm{RDF}-\mathrm{RULEMIN} \subseteq(G, \mathcal{R})$ be the following decision problem: INPUT: An RDF graph $G$ and a set $\mathcal{R}$ of $R D F$ rules.

QUESTION: Does there exist $\mathcal{R}^{\prime} \subset \mathcal{R}$ s.t. $C l_{\mathcal{R}^{\prime}}(G)=C l_{\mathcal{R}}(G)$ ?

For the case that the set of rules is b-bounded, we can pinpoint the complexity of the problem to NP.

Theorem 2. For a set $\mathcal{R}$ of b-bounded rules (for fixed b), the problem RDFRULEMIN $=(G, \mathcal{R})$ is NP-complete while RDF-RULEMIN $\subseteq(G, \mathcal{R})$ is in PTIME.

Proof. The hardness is shown by reduction from the 3-Colorability problem. The RDF graph $G$ is built over the URIs $U=\{0,1,2\}$ in subject and object positions. $G$ contains triples of the form $i$ e $j$ for all value combinations $i, j \in U$ with $i \neq j$. $\mathcal{R}$ contains a single rule which generates an encoding $X_{\alpha} e X_{\beta}$ (with blank nodes $\left.X_{\alpha}, X_{\beta}\right)$ for each edge $\left(v_{\alpha}, v_{\beta}\right)$ of the graph to be 3-colored. This rule is redundant iff a valid 3-coloring exists, i.e., iff the triples $X_{\alpha} e X_{\beta}$ can be mapped into $\{i$ e $j \mid i \neq j\}$.

For the membership, note that it suffices to compare the closure of $G$ under $\mathcal{R}$ with the closure of $G$ under every subset of $\mathcal{R}$ missing exactly one rule. In the b-bounded case, the closure can be computed efficiently. Hence, we get PTIMEmembership for RDF-RULEMIN $\subseteq$ and NP-membership for RDF-RULEMIN ${ }^{\models}$ (the NP-computation is needed only for the entailment check).

Theorem 3. For arbitrary rules, RDF-RULEMIN $\subseteq(G, \mathcal{R})$ is coNP-hard and in $\Delta_{2}^{P}$ while RDF-RULEMIN ${ }^{\circ}(G, \mathcal{R})$ is NP-hard, coNP-hard, and in $\Delta_{2}^{P}$.

Proof. The $\Delta_{2}^{P}$ upper bound is due to the fact that computing the closure under a set of arbitrary rules requires an NP-oracle (to check if a rule is applicable). The NP-hardness of RDF-RULEMIN ${ }^{\prime}(G, \mathcal{R})$ carries over from Theorem 2. The coNP-hardness of both problems is shown by a straightforward reduction from the co-problem of 3-Colorability: $\mathcal{R}$ contains a single rule whose body encodes the graph to be 3-colored. This rule is redundant iff no 3-coloring exists.

In order to reduce the complexity of the problems RDF-RULEMIN $\subseteq(G, \mathcal{R})$ and RDF-RULEMIN ${ }^{=}(G, \mathcal{R})$, one could seek for approximations of those problems. In fact, one option is to check for redundant rules in the set $\mathcal{R}$ of given Datalog rules; or whether some rule is subsumed by another rule from $\mathcal{R}$. The first problem is known to be tractable while the test for rule subsumption is NP-complete (see [17]). The latter result can be shown to hold also for rules of bounded arity (which we deal with here); but becomes tractable in the case of b-bounded rules. Further methods (e.g., folding and unfolding of rules) are well understood for logic programs (see [18]), and could also apply to our domain. An in-depth analysis how to use those results in our setting is left for future work.

\section{Minimisation w.r.t. Queries}

Another variant of the RDF graph and rule minimisation problems is to guarantee completeness only w.r.t. a given set of queries. We restrict ourselves here to (unions of) conjunctive queries (CQs resp. UCQs). Such a minimisation is of high interest, e.g. when importing data into an RDF Store that provides a narrow query interface only. Formally, we get the following problems: 
10

\begin{tabular}{|l|l|c|c|c|}
\hline & & $\mathcal{Q}$ body-bb (a) & $\mathcal{Q}$ head-bb (b) & $\mathcal{Q}$ arb. (c) \\
\hline$(1)$ & $\mathcal{R}$ arb., $\mathcal{C}$ arb. & $\Sigma_{3}^{P}$-complete & $\Sigma_{3}^{P}$-complete & $\Sigma_{3}^{P}$-complete \\
\hline$(2)$ & $\mathcal{R}$ arb., $\mathcal{C}$ bb & $\mathrm{NP} / \Delta_{2}^{P}$ & $\mathrm{NP} / \Delta_{2}^{P}$ & $\Sigma_{3}^{P}$-complete \\
\hline$(3)$ & $\mathcal{R}$ arb., $\mathcal{C}$ fixed & $\mathrm{NP} / \Delta_{2}^{P}$ & $\mathrm{NP} / \Delta_{2}^{P}$ & $\Sigma_{3}^{P}$-complete \\
\hline$(4)$ & $\mathcal{R}$ arb., $\mathcal{C}=\emptyset$ & $\mathrm{NP} / \Delta_{2}^{P}$ & $\mathrm{NP} / \Delta_{2}^{P}$ & $\Pi_{2}^{P}$-complete \\
\hline$(5)$ & $\mathcal{R}$ bb., $\mathcal{C}$ arb. & $\Sigma_{3}^{P}$-complete & $\Sigma_{3}^{P}$-complete & $\Sigma_{3}^{P}$-complete \\
\hline$(6)$ & $\mathcal{R}$ bb, $\mathcal{C}$ bb & $\mathrm{NP}$-complete & $\mathrm{NP} / \Delta_{2}^{P}$ & $\Sigma_{3}^{P}$-complete \\
\hline$(7)$ & $\mathcal{R}$ bb, $\mathcal{C}$ fixed & $\mathrm{NP}$-complete & $\mathrm{NP} / \Delta_{2}^{P}$ & $\Sigma_{3}^{P}$-complete \\
\hline$(8)$ & $\mathcal{R}$ bb, $\mathcal{C}=\emptyset$ & in $\mathrm{P}$ & $\mathrm{NP} / \Delta_{2}^{P}$ & $\Pi_{2}^{P}$-complete \\
\hline$(9)$ & $\mathcal{R}$ fixed, $\mathcal{C}$ arb. & $\Sigma_{3}^{P}$-complete & $\Sigma_{3}^{P}$-complete & $\Sigma_{3}^{P}$-complete \\
\hline$(10)$ & $\mathcal{R}$ fixed, $\mathcal{C}$ bb & $\mathrm{NP}$-complete & $\mathrm{NP} / \Delta_{2}^{P}$ & $\Sigma_{3}^{P}$-complete \\
\hline$(11)$ & $\mathcal{R}$ fixed, $\mathcal{C}$ fixed & $\mathrm{NP}$-complete & $\mathrm{NP} / \Delta_{2}^{P}$ & $\Sigma_{3}^{P}$-complete \\
\hline$(12)$ & $\mathcal{R}$ fixed, $\mathcal{C}=\emptyset$ & in $\mathrm{P}$ & $\mathrm{NP} / \Delta_{2}^{P}$ & $\Pi_{2}^{P}$-complete \\
\hline \hline$(\mathrm{I}$ ) & $\mathcal{R}$ arb. & coNP $/ \Delta_{2}^{P}$ & $\mathrm{coNP}+\mathrm{NP} / \Delta_{2}^{P}$ & $\Pi_{2}^{P}$-complete \\
\hline$($ II. $)$ & $\mathcal{R}$ bb. & in $\mathrm{P}$ & $\mathrm{NP} / \Delta_{2}^{P}$ & $\Pi_{2}^{P}$-complete \\
\hline
\end{tabular}

Table 2. The complexity of MINI-RDF $\subseteq, C Q$ (1-12) and RDF-RULEMIN $\subseteq, C Q$ (I. - II.) w.r.t. input parameters ("bb" stands for "b-bounded", and "arb." for "arbitrary").

Definition 5. MINI-RDF $\subseteq, C Q(G, \mathcal{R}, \mathcal{C}, \mathcal{Q})$ is the following decision problem: INPUT: An RDF graph $G$, a set $\mathcal{R}$ of $R D F$ rules, a set $\mathcal{C}$ of tgds ( $G$ satisfies $\mathcal{C})$, and a set $\mathcal{Q}$ of $C Q s$.

QUESTION: Is there $a G^{\prime} \subset G$ s.t. (1) for every $q \in \mathcal{Q}$, the answers to $q$ over $C l_{\mathcal{R}}(G)$ coincide with the answers to $q$ over $C l_{\mathcal{R}}\left(G^{\prime}\right)$ and (2) $G^{\prime}$ satisfies $\mathcal{C}$ ?

Definition 6. RDF-RULEMIN $\subseteq, C Q(G, \mathcal{R}, \mathcal{Q})$ is the following decision problem: INPUT: An RDF graph $G$, a set $\mathcal{R}$ of $R D F$ rules, and a set $\mathcal{Q}$ of $C Q s$.

QUESTION: Is there an $\mathcal{R}^{\prime} \subset \mathcal{R}$ s.t. for every $q \in \mathcal{Q}$, the answers to $q$ over $C l_{\mathcal{R}}(G)$ coincide with the answers to $q$ over $C l_{\mathcal{R}^{\prime}}(G)$ ?

First, we observe that all hardness results from Sections 3 and 4 carry over to the CQ-variants. To see this, we note that, for instance, MINI-RDF $\subseteq$ corresponds to the special case of MINI-RDF $\subseteq, C Q$ where $\mathcal{Q}=\{\{S P O\} \rightarrow \operatorname{ans}(S, P, O)\}$.

Analogously to the various settings studied in the previous sections resulting from different restrictions on $\mathcal{C}$ and $\mathcal{R}$, we also study three settings of the CQvariants of these problems by considering $\mathcal{Q}$ to be body-b-bounded, head-bbounded, or unrestricted, respectively. We thus get the following complexity results.

Theorem 4. For MINI-RDF $\subseteq, C Q$, the complexity w.r.t. different assumptions on the input (arbitrary, b-bounded or fixed rule set; arbitrary, b-bounded, fixed, or no constraints; body-b-bounded, head b-bounded, or arbitrary CQs) is as depicted in Table 2, rows (1) - (12). Likewise, the complexity of RDF-RULEMIN $\subseteq, C Q$ is depicted in Table 2 , rows (I) - (II).

Thereby (co-) NP / $\Delta_{2}^{P}$ denotes the lower bound / upper bound for the complexity. We write coNP $+\mathrm{NP} / \Delta_{2}^{P}$ if both, coNP- and NP-hardness hold. All lower bounds hold even if $Q$ consists of a single $C Q$. Likewise, all upper bounds hold even if $\mathcal{Q}$ is a set of $U C Q s$. 
Obviously, body-b-bounded (U)CQs are a special case of head-b-bounded (U)CQs, which in turn are a special case of arbitrary (U)CQs. By combining this observation with Lemma 2, to prove Theorem 4, it suffices to show membership for the entries (6a), (8a), (2b), (1c), (4c) as well as (Ia), (IIb), and (IIc) in Table 2, and hardness for (11a), (12b), (11c), (12c) as well as (IIa), (Ib), and (Ic). Due to space restrictions, we only give a rough sketch of the intuition of these results. All proofs are worked out in detail in the full version [12].

Membership of the most general case (1c) is shown by considering the following algorithm: guess a subset $G^{\prime} \subset G$ and check with $\Pi_{2}^{P}$-oracles if $G^{\prime}$ satisfies $\mathcal{C}$ and if $q(\hat{G})=q\left(\hat{G}^{\prime}\right)$, where $\hat{G}=C l_{\mathcal{R}}(G)$, resp. $\hat{G}^{\prime}=C l_{\mathcal{R}}\left(G^{\prime}\right)$. Moreover, the closures $\hat{G}$ and $\hat{G}^{\prime}$ can be computed in $\Delta_{2}^{P}$, since they are subsets of $A D^{3}$.

The other columns contain potentially easier settings because of the restrictions on the queries, while the other rows are potentially easier because of restrictions on $\mathcal{R}$ and $\mathcal{C}$. In particular, if no constraints are present, it suffices to check the "direct" subsets $G^{\prime}=G \backslash\{t\}$ for each $t \in G$. Thus the non-deterministic guess of $G^{\prime} \subset G$ is no longer needed. By the same token, rule minimisation is not harder than $\Pi_{2}^{P}$, since we only need to check the direct subsets $\mathcal{R}^{\prime}=\mathcal{R} \backslash\{r\}$. If the queries are head-b-bounded, then there are at most polynomially many candidates for answer-tuples. Hence, to answer a query $q$ over two different RDF graphs is feasible in $\Delta_{2}^{P}$ (rather than $\Pi_{2}^{P}$ ). For body-b-bounded queries, the answers to a query $q$ over an RDF graph can even be computed in PTIME.

Turning to the lower bounds, the NP-hardness for (11a) follows immediately from the above remark that the hardness results of MINI-RDF $\subseteq$ carry over. (12b) differs from the previous setting by allowing more expressive queries, but no constraints (which, for MINI-RDF $\subseteq$, leads to tractability). However, the NPhardness of this case follows immediately from the coNP-hardness of checking if an RDF graph is lean [15] and defining $\mathcal{Q}=\{G \rightarrow$ ans ()$\}$. The hardness for (11c) is shown by reduction from $\mathrm{QSAT}_{3}$. Its main idea is, given a formula $F=$ $\exists \boldsymbol{x}_{1} \forall \boldsymbol{y}_{1} \exists \boldsymbol{x}_{2} \phi$, to define a CQ $q$ and a graph $G$ such that every homomorphism $\tau: \operatorname{body}(q) \rightarrow G$ defines a truth assignment on the variables in $F$. (The proof allows even $\mathcal{R}=\emptyset$.) Thereby $q$ outputs the values of this truth assignment on $\boldsymbol{y}_{1} . G$ is further chosen in such a way that $q(G)$ contains an encoding of all possible truth assignments on $\boldsymbol{y}_{1}$. The constraints in $\mathcal{C}$ are such that over every proper subgraph $G^{\prime} \subset G$ that satisfies $\mathcal{C}$, every homomorphism from $\operatorname{body}(q)$ to $G^{\prime}$ now encodes truth assignments that actually satisfy $\phi$. At the same time, the assignment on $\boldsymbol{x}_{1}$ is already defined by the choice of $G^{\prime}$. Hence, if $q\left(G^{\prime}\right)$ also contains encodings for all possible truth assignments on $\boldsymbol{y}_{1}$, this means that $F$ is indeed satisfied. For $\mathcal{C}=\emptyset$, we only get $\Pi_{2}^{P}$-hardness since we can no longer express that valid choices for $G^{\prime}$ encode a truth assignment on $\boldsymbol{x}_{1}$.

For the rule minimisation, the $\Pi_{2}^{P}$-hardness is shown similarly to the $\Pi_{2}^{P}$ hardness in case (12c). In case of (head-/body-)b-bounded queries, the answers to the queries can no longer produce all possible truth assignments on $\boldsymbol{y}_{1}$. Hence, we can only prove NP- and coNP-hardness, respectively, in cases (Ia) and (IIa).

\subsection{Beyond Conjunctive Queries - SPARQL}

RDF minimization w.r.t. (unions of) conjunctive queries could be extended to more expressive query languages. Actually, it can be checked that all upper 
bounds proved in Section 5 are still valid if the CQs are allowed to contain negation in the body. In particular, the complexity of the problems considered here does not go beyond $\Sigma_{3}^{P}$ for this kind of extension, cf. [12]. In contrast, if we allow arbitrary non-recursive datalog queries with negation (a query language which - as well known - covers all of SPARQL [19]), then the complexity of the problems considered here will be dominated by the complexity of query evaluation, which is PSPACE-complete in this case, see [20]. We leave a more fine-grained analysis of different fragments of SPARQL for future work.

\section{Problem Variations}

In this section, we discuss some further problems which are variations of or strongly related to the problems studied in the previous sections. We start by a variation of the graph minimisation problem. But now we ask if $G$ can be replaced by a subgraph $G^{\prime}$ whose size is bounded by some given bound $k$ (rather than an arbitrary subgraph $G^{\prime} \subset G$ ). Formally, we study the following problem.

Definition 7. Let MINI-RDF ${ }^{\text {card }}(G, \mathcal{R}, \mathcal{C}, k)$ be the following decision problem: INPUT: An RDF graph $G$, a set $\mathcal{R}$ of $R D F$ rules, a set $\mathcal{C}$ of tgds and integer $k$. QUESTION: Does there exist a subgraph $G^{\prime} \subset G$ with $\left|G^{\prime}\right| \leq k$, s.t. $G^{\prime}$ satisfies $\mathcal{C}$ and $G \subseteq C l_{\mathcal{R}}\left(G^{\prime}\right)$ ?

It can be easily verified that for all cases in Table 1 that are at least NP-hard, the complexity for MINI-RDF ${ }^{\text {card }}$ does not change. Intuitively, this is because the nondeterministic algorithms for solving these problems all start with "guess a subgraph $G^{\prime} \subset G^{\prime}$ ", which can be easily changed to "guess a subgraph with at most $k$ triples". Therefore, the only two interesting cases are MINI-RDF $\subseteq$ with a b-bounded or fixed set $\mathcal{R}$ and no constraints, as they can be decided in PTIME. We show that for MINI-RDF ${ }^{\text {card }}$, the complexity goes up to NP-completeness.

Theorem 5. The problem MINI-RDF ${ }^{\text {card }}(G, \mathcal{R}, \mathcal{C}, k)$ is $\mathrm{NP}$-complete if $\mathcal{C}=\emptyset$ and $\mathcal{R}$ is either considered as fixed or a set of b-bounded rules (for fixed $b$ ).

Proof. The hardness proof is by reduction from the Vertex Cover problem. We give the basic ideas of this reduction. Given some graph $G=(V, E)$, the RDF graph $G^{r d f}$ contains one distinct triple for every $v \in V$. The intuition is that the subset of those triples contained in a valid subgraph $G^{\prime} \subset G^{r d f}$ describes a vertex cover. We further have three rules, one that (given $G^{\prime} \subset G$ ) adds all edges covered by the remaining vertices in $G^{\prime}$, one that (by repeated application) checks whether all edges are covered, and finally one rule that, if indeed all edges are covered, allows to restore the vertices from $G^{r d f} \backslash G^{\prime}$. To allow to express according rules, $G^{r d f}$ contains triples encoding further information (like e.g. neighbourhood of vertices and edges). But as they cannot be derived by any rule, they must remain unchanged in any valid $G^{\prime} \subset G^{r d f}$. Further, their number (say $K$ ) only depends on $G$, such that there exists a vertex cover of size $k$ iff there exists a valid $G^{\prime} \subset G^{r d f}$ of size $K+k$.

Next we want to identify the sources of the complexity of MINI-RDF ${ }^{\models}$ and MINI$\mathrm{RDF} \subseteq$ for the cases where $\mathcal{C}$ is allowed to contain arbitrary tgds. We show that the complexity is independent of the rules, but arises mainly from the question whether there exists some non-empty subgraph that satisfies all constraints. 
Theorem 6. Let $G$ be an $R D F$ graph and $\mathcal{C}$ a set of tgds. Deciding whether there exists some $\emptyset \neq G^{\prime} \subset G$ s.t. $G^{\prime}$ satisfies $\mathcal{C}$ is $\Sigma_{3}^{P}$-complete.

Proof. Membership follows from Theorem 1. Hardness is shown by a modification of the reduction given in the proof of Lemma 3. We give the intuition of these modifications. In the aforementioned proof, the intuitive meaning of the rules, together with the requirement $G \subseteq C l_{\mathcal{R}}\left(G^{\prime}\right)$, was that for each $v_{i} \in \boldsymbol{x}_{1}$, either $\left\{\begin{array}{lll}v_{i} & q_{1} & a_{01}\end{array}\right\}$ or $\left\{\begin{array}{llll}v_{i} & q_{1} & a_{10}\end{array}\right\}$ has to remain in the subgraph $G^{\prime}$. However, this can be also formulated as a constraint. By introducing an additional triple for every $v_{i} \in \boldsymbol{x}_{\mathbf{1}}$ (e.g. $\left\{v_{i}\right.$ opt $\left.v_{i}\right\}$ ) that is enforced to be contained in any non-empty subgraph, the tgd $\{V$ opt $V\} \Rightarrow\left\{V q_{1} A\right\}$ does the job.

From the (full) proof of Lemma 4, it follows that for MINI-RDF", one source of the NP-hardness is just to decide the entailment. However, similarly to the last theorem, we can show that for b-bounded tgds, just testing for the existence of a valid subgraph already contains the full hardness too.

Theorem 7. Let $G$ be an $R D F$ graph and $\mathcal{C}$ a set of b-bounded tgds. Deciding whether there exists some $\emptyset \neq G^{\prime} \subset G$ s.t. $G^{\prime}$ satisfies $\mathcal{C}$ is $\mathrm{NP}$-complete.

Proof. Membership follows from Theorem 1. Hardness is shown by reduction from the SAT problem. The reduction is very similar to the one of Lemma 4, only that all the implicit information about which triples must not be removed from $G$ (expressed by not providing rules to derive them) now have to be made explicit as tgds. This however no longer allows for a fixed set of tgds, but makes the number of tgds dependent on $F$.

Recall that tgds generalize (safe) datalog rules by allowing existential quantification and conjunctions in the head. In other words, datalog rules are an important special case of tgds - referred to as full tgds in the information integration literature. Below, we show that restricting the constraints to full tgds pushes the $\Sigma_{3}^{P}$-completeness results from Theorems 1 and 6 down to $\Sigma_{2}^{P}$.

Theorem 8. The problems $\mathrm{MINI}-\mathrm{RDF}^{\models}=(G, \mathcal{R}, \mathcal{C})$ and $\operatorname{MINI-RDF} \subseteq(G, \mathcal{R}, \mathcal{C})$ are $\Sigma_{2}^{P}$-complete if $\mathcal{C}$ is a set of full tgds. $\Sigma_{2}^{P}$-completeness even holds for fixed $\mathcal{R}$.

Likewise, let $G$ be an RDF graph and $\mathcal{C}$ a set of full tgds. Deciding whether there exists some $\emptyset \neq G^{\prime} \subset G$ s.t. $G^{\prime}$ satisfies $\mathcal{C}$ is $\Sigma_{2}^{P}$-complete.

Proof. The $\Sigma_{2}^{P}$-membership is established by the same algorithm as the $\Sigma_{3}^{P}$ membership in case of unrestricted tgds according to Theorem 1. However, by the restriction to full tgds, we now only need a coNP-oracle (rather than $\Pi_{2}^{P}$ ) for checking that the tgds are satisfied. The $\Sigma_{2}^{P}$-hardness is shown via reduction from $\mathrm{QSAT}_{2}$ by using similar ideas as in the $\Sigma_{3}^{P}$-hardness proof in Lemma 3.

\subsection{General RDF Rules vs. Datalog Rules}

We now show that the complexity of the investigated problems remains unchanged by allowing additional predicates uri(.), blank(.), lit(.) to restrict the type of a value in a Datalog rule, that is, allowing general RDF rules as defined in Section 2. Note that for every $x \in U \cup B \cup L$ occurring in some RDF-graph $G$ 
(i.e. for every element of the active domain) it can be easily recognised whether it belongs to $U, B$ or $L$ : This could be either decided using syntactic criteria, or by a lookup in $U, B$ and $L$ (although those sets are supposed to be countable infinite, one can assume that $U_{G}, B_{G}$ and $L_{G}$, i.e. the elements of the active domain, are the "first" elements of these sets). Therefore, determining the type of some element requires at most polynomial time in the size of $G$. Therefore, for every element $x$ of the active domain of $G$, we create a ground atom $B_{t}(x), U_{t}(x)$ or $L_{t}(x)$, depending on the type of $x$. By encoding an atom blank $(X)$ as triple $\{X$ blank $X\}$ in $G$, we can make this information available for rule application without increasing the complexity of the problem.

The same argument allows us to overcome the problem that the closure w.r.t. a rule set $\mathcal{R}$ contains invalid RDF triples (containing e.g. a blank node in a predicate position). Depending on whether invalid triples are allowed in intermediate results or not, we can pursue one of the following two strategies: (i) in a postprocessing step, we can check for every triple in $\mathcal{R}(G)$ whether it is valid or not. In the latter case, it is removed; (ii) if invalid triples should also be excluded from any intermediate results, then the rules can be (automatically) augmented by at most 2 additional predicates in the rule body, $\operatorname{urib}(A)$ and $\operatorname{uri}(B)$, assuming that the rule head is $\{A B C\}$. The predicate $u r i b($.$) can be easily defined from uri(.)$ and $\operatorname{blank}($.$) by e.g. \{\operatorname{uri}(X)\} \Rightarrow\{\operatorname{urib}(X)\}$ and $\{\operatorname{blank}(X)\} \Rightarrow\{\operatorname{urib}(X)\}$. This is similar to variations of rules (2)-(4) in Section 1, where the filter conditions guaranteed valid intermediate triples.

\section{Conclusion}

We proved a collection of complexity results for minimisation problems over RDF graphs where we considered various restrictions on the rules and tgds. One such restriction was b-boundedness [11]. We note that this restriction can be relaxed by bounding not necessarily the size of the rules (or tgds) but only the maximal number of blank nodes occurring in the rules (or tgds) - in the Datalog world, Vardi [21] showed that such a restriction decreases complexity. We further discussed how the complexity of the problem increases if one requires completeness only with respect to a given set of conjunctive queris (CQs). Notably, if the CQs are restricted to have bounded head arity, while providing additional minimisation potential, the problem becomes only mildly harder.

The minimisation problems considered here are driven by practical needs to represent RDF data compactly or tailor them to engines supporting different rule sets. Our results also provide a basis for eliminating redundancies in existing practically relevant rule sets, such as OWL2RL [8]. We believe that our results will gain even more relevance with the advent of novel standards such as the W3C rule interchange format (RIF) which will allow one to enrich RDFS and OWL with Web-publishable custom rule sets [22].

As future work, our investigations should be further extended in several directions such as a more fine-grained analysis of SPARQL fragments when redundancy w.r.t. queries is considered, for instance well-designed SPARQL queries [20]. Moreover, we plan to cast the obtained results into practical algorithms to "compress" RDF graphs and rule sets, investigate related relevant problems such as "trading" triples for rules, or vice versa, and experimentally 
evaluating effects of such transformations on query answering with dynamic inference such as sketched in [2].

\section{References}

1. Hogan, A., Decker, S.: On the ostensibly silent 'W' in OWL 2 RL. In: Proc. RR'09. Volume 5837 of LNCS., Springer (2009) 118-134

2. Ianni, G., Krennwallner, T., Martello, A., Polleres, A.: Dynamic querying of massstorage RDF data with rule-based entailment regimes. In: Proc. ISWC'09. Volume 5823 of LNCS., Springer (2009) 310-327

3. Muñoz, S., Pérez, J., Gutiérrez, C.: Minimal deductive systems for RDF. In: Proc. ESWC'07. Volume 4519 of LNCS., Springer (2007) 53-67

4. Grosof, B.N., Horrocks, I., Volz, R., Decker, S.: Description Logic Programs: Combining Logic Programs with Description Logics. In: Proc. WWW'03. (2003) 48-57

5. de Bruijn, J., Polleres, A., Lara, R., Fensel, D.: OWL ${ }^{-}$. WSML D20.1v0.2 (2005)

6. ter Horst, H.J.: Completeness, decidability and complexity of entailment for RDF Schema and a semantic extension involving the OWL vocabulary. J. Web Sem. 3(2-3) (2005) 79-115

7. Hogan, A., Harth, A., Polleres, A.: Scalable authoritative OWL reasoning for the web. International Journal on Semantic Web and Information Systems 5(2) (2009)

8. Motik, B., Grau, B.C., Horrocks, I., Wu, Z., Fokoue, A., Lutz, C.: OWL 2 Web ontology language profiles (October 2009) W3C Recommendation.

9. Hayes, P.: RDF semantics. Technical report, W3C (February 2004) W3C Recommendation.

10. Lausen, G., Meier, M., Schmidt, M.: SPARQLing constraints for RDF. In: Proc. EDBT'08, ACM Press

11. Meier, M.: Towards Rule-Based Minimization of RDF Graphs under Constraints. In: Proc. RR'08. Volume 5341 of LNCS., Springer (2008) 89-103

12. Pichler, R., Polleres, A., Skritek, S., Woltran, S.: Minimizing RDF graphs under rules and constraints revisited. Technical report, DERI (April 2010) Available at http://www .deri.ie/fileadmin/documents/DERI-TR-2010-04-23.pdf.

13. Beckett, D., Berners-Lee, T.: Turtle - Terse RDF Triple Language (January 2008) W3C Team Submission, http://www.w3.org/TeamSubmission/turtle/.

14. Ullman, J.D.: Principles of Database and Knowledge Base Systems. Computer Science Press, New York, NY, USA (1989)

15. Gutierrez, C., Hurtado, C., Mendelzon, A.: Foundations of semantic web databases. In: Proc. 04, ACM (2004) 95-106

16. Eiter, T., Faber, W., Fink, M., Woltran, S.: Complexity results for answer set programming with bounded predicate arities and implications. Ann. Math. Artif. Intell. 51(2-4) (2007) 123-165

17. Eiter, T., Fink, M., Tompits, H., Traxler, P., Woltran, S.: Replacements in nonground answer-set programming. In: Proc. KR'06, AAAI Press (2006) 340-351

18. Pettorossi, A., Proietti, M.: Transformation of logic programs. In Gabbay, D.M., Hogger, C.J., Robinson, J.A., eds.: Handbook of Logic in Artificial Intelligence and Logic Programming. Volume 5., Oxford University Press (1998) 697-787

19. Angles, R., Gutierrez, C.: The expressive power of SPARQL. In: Proc. ISWC 2008. Volume 5318 of LNCS., Springer (2008) 114-129

20. Pérez, J., Arenas, M., Gutierrez, C.: Semantics and complexity of SPARQL. ACM Trans. Database Syst. 34(3) (2009)

21. Vardi, M.: On the complexity of bounded-variable queries. In: Proc. PODS'95, ACM Press (1995) 266-276

22. de Bruijn, J.: RIF RDF and OWL Compatibility (May 2010) W3C Proposed Recommendation, http://www.w3.org/TR/2010/PR-rif-rdf-owl-20100511/. 